Research Paper

\title{
The Association of Polymorphisms in Base Excision Repair Genes with Ovarian Cancer Susceptibility in Chinese Women: A Two-Center Case-Control Study
}

Mingyao Zhang1\#, Zhiguang Zhao#, Sailing Chen ${ }^{1}$, Zongwen Liang ${ }^{1}$, Jiawei Zhu' ${ }^{1}$, Manman Zhao ${ }^{1}$, Chaoyi $\mathrm{Xu}^{1}$, Jing $\mathrm{He}^{3}$, Ping Duan ${ }^{1 凶}$ and Anqi Zhang ${ }^{1 凶}$

1. Department of Obstetrics and Gynecology, The Second Affiliated Hospital and Yuying Children's Hospital of Wenzhou Medical University, Wenzhou 325027, Zhejiang, China.

2. Department of Pathology, The Second Affiliated Hospital and Yuying Children's Hospital of Wenzhou Medical University, Wenzhou 325027, Zhejiang, China.

3. Department of Pediatric Surgery, Guangzhou Institute of Pediatrics, Guangzhou Women and Children's Medical Center, Guangzhou Medical University, Guangzhou 510623, Guangdong, China.

\#These authors contributed equally to the work.

$\triangle$ Corresponding authors: Anqi Zhang, E-mail: angel19911014@gmail.com \& Ping Duan, E-mail: dppddpp@wmu.edu.cn. Department of Obstetrics and Gynecology, The Second Affiliated Hospital and Yuying Children's Hospital of Wenzhou Medical University, 109 West Xueyuan Road, Wenzhou 325027, Zhejiang, China. Tel and Fax: (0577)88816381.

(c) The author(s). This is an open access article distributed under the terms of the Creative Commons Attribution License (https://creativecommons.org/licenses/by/4.0/). See http:/ /ivyspring.com/terms for full terms and conditions.

Received: 2020.06.25; Accepted: 2020.10.11; Published: 2021.01.01

\begin{abstract}
Base excision repair (BER) acts upon the most important mechanism of the DNA repair system, protecting DNA stability and integrity from the mutagenic and cytotoxic effects. Multiple researches have indicated that single-nucleotide polymorphisms (SNPs) in the BER-related gene may be associated with the susceptibility of ovarian cancer. However, the results are controversial. In this two-center case-control study, 19 potentially functional SNPs in six BER-related genes (hOGGI, APEI, PARPI, FENI, LIG3 and XRCCI) was genotyped in 196 ovarian cancer cases and 272 cancer-free controls. And, their associations with ovarian cancer risk were assessed by unconditional logistic regression analyses. We found that PARPI rs8679 and hOGGI rs293795 polymorphisms were associated with a decreased risk of ovarian cancer under dominant model (adjusted $\mathrm{OR}=0.39,95 \% \mathrm{Cl}=0.17-0.90, P=0.026$; and adjusted $\mathrm{OR}=0.36,95 \% \mathrm{Cl}=0.13-0.99, P=0.049$, respectively). Stratification analysis demonstrated that this association was more pronounced in the subgroups of lower BMI and patients with early menarche and serous carcinoma. Moreover, LIG3 rs4796030 AA/AC variant genotypes performed an increased risk of ovarian cancer under recessive model (adjusted $\mathrm{OR}=1.54,95 \% \mathrm{Cl}=1.01-2.35, P=0.046$ ), especially in the subgroups of higher BMI, early clinic stage and the carcinoma at the left. These results suggested that PARPI, hOGGI and LIG3 polymorphisms might impact on the risk of ovarian cancer. However, more researches with larger and different ethnic populations are warranted to support our findings.
\end{abstract}

Key words: BER; DNA repair; ovarian cancer; susceptibility; polymorphism

\section{Introduction}

Ovarian cancer is the main cause of death in gynecological malignancy according to global cancer statistics in 2018, with an estimated 184,799 deaths worldwide [1]. The common risk factors of that result in extremely poor 5-year survival, such as asymptomatic characteristics and the lack of early detection strategies, have become an immediate problem to be solved for ovarian cancer $[2,3]$. Thus, ovarian cancer remains a great burden and challenge for the affected family and public health.

Numerous studies have indicated that the risk of ovarian cancer was related to $B R C A 1$ and $B R C A 2$ genes. Marc D. et al. [4] found that BRCA1/2 mutations may ultimately impact ovarian cancer 
mortality. Adrianna et al. [5] demonstrated significantly increased APC rs11954856 and rs351771 frequencies in Polish women with ovarian cancer. However, several genetic studies published recently had suggested that the risk of ovarian cancer was related to the mutations and polymorphic variants in DNA repair genes $[6,7]$. DNA repair mechanism mainly include direct repair, base excision repair (BER), nucleotide excision repair (NER) and mismatch repair (MMR) [8]. The primary DNA damage repair pathway, BER, is responsible for the modification of the most common forms of DNA damage, such as spontaneous hydrolytic, oxidative and alkylative lesions and single-strand breaks [9]. Although considerable genetic studies demonstrated that the deficiency of BER related genes would influence on ovarian cancer risk, the real relationship between both of them remained controversial [10].

BER processes can be divided into the following three basic steps: damage recognition/strand scission, gap tailoring and gap filling/nick ligation [11]. Based on different types of base lesions, either of two subways (short patch or long patch BER) will be initiated by corresponding lesion-specific DNA glycosylase [12]. 8-oxoguanine glycosylase (hOGG1) acts as one of the most important bifunctional glycosylases, which cannot only excise the substrate base but also incises DNA 3' to the damage site via an intrinsic AP lyase activity [13]. Meanwhile, apurinic endonuclease 1 (APE1) removes the resulting 3 '-obstructive termini and prepares for next polymerization and/or nick ligation [14]. Differing from the classical initial step, however, the singlestrand break repair (SSBR) depends on poly (ADPribose) polymerase 1 (PARP1) to recognize the damage and recruit other protein to the damage site [15]. In addition, flap endonuclease (FEN1) was also included in our study for its gap-tailoring properties in the DNA ends [16]. DNA ligase 1 (LIG1) and the complex of DNA ligase 3 (LIG3)/X-ray repair cross-complementing 1 (XRCC1) exhibit similar performance of nick ligation, but we only selected LIG3/XRCC1 as the investigated biomarker for its unique character of collecting proteins at the damaged site $[17,18]$. In the current study, we analyze the associations between 19 SNPs of six BER-related genes (hOGG1, APE1, PARP1, FEN1, LIG3 and XRCC1) and ovarian cancer risk by genotyping method in 196 patients and 272 controls.

\section{Materials and Methods}

\section{Patients and controls}

In this hospital-based case-control study, 196 ovarian cancer patients were newly diagnosed and histopathologically confirmed in two different institutions of the Second Affiliated Hospital and Yuying Children's Hospital and the First Affiliated Hospital of Wenzhou Medical University (WMU) from February 2007 to March 2018. Additionally, we selected 272 cancer-free women who participated in the routine physical examination of the Second Affiliated Hospital and Yuying Children's Hospital of WMU. All of them were frequency-matched to cases on age ( \pm 2 years) and race/ethnicity, and reconfirmed without any personal tumor history or family tumor history. All people included in the present research had signed a written informed consent. The demographic data and environmental exposure factors, such as age, BMI, menarche, menopause, number of pregnancies, were collected from each participant. Clinical and pathological information were further extracted from the patients' electronical database, including pathologic classification (WHO 2014), histology grade, FIGO stage (International Federation of Gynecology and Obstetrics), tumor size (the largest tumor diameter of the primary tumor) and anatomic neoplasm subdivision. However, the patients who were diagnosed without histology grade would be categorized as a single group of "undermined". And, some patients with some rare pathological classification were included in the "others" pathological group. The research was approved by the Second Affiliated Hospital and Yuying Children's Hospital of WMU. Our study was conducted following the Declaration of Helsinki, and participants or guardians were required to sign informed consent forms. Blood samples were obtained from cases before receiving radiotherapy or chemotherapy.

\section{SNP selection and genotyping}

The SNPs were selected from the NCBI dbSNP database (http://www.ncbi.nlm.nih.gov/projects/ SNP) and the International HapMap Project database (http://hapmap.ncbi.nlm.nih.gov/) based on the following criteria: (1) located at the coding sequence, the $5^{\prime}$ near gene, 5' -untranslated region (UTR), 3' UTR and $3^{\prime}$ near gene, (2) minor allele frequency (MAF) of at least $5 \%$ in Chinese populations reported in HapMap, (3) low linkage disequilibrium (LD) between SNPs with an $\mathrm{r}^{2}$ threshold of $<0.8$, and (4) predicted SNPs potential function using the SNP function prediction (FuncPred) software (https:// snpinfo.niehs.nih.gov/snpinfo/snpfunc.html). As a result, a full list containing 19 selected SNPs was presented with potential function in Supplemental Table S1 [19]. 
For all cases, the DNA genomic was extracted from paraffin-embedded tissue using TIANquick FFPE DNA Kit (Qiagen Inc., Valencia, CA), while the DNA genomic of all controls were extracted from the peripheral blood specimens using the TIANamp Blood DNA Kit (TianGen Biotech Co. Ltd.). DNA purity and concentration were measured by a UV spectrophotometer (Nano Drop Technologies, Inc., Wilmington, DE).

According to standard protocols, the genotyping of 19 selected SNPs was performed with a TaqMan real-time PCR assay in ABI Prism 7900HT genetic detection system (Applied Biosystems Inc., USA) [20-22]. To confirm the accuracy of the genotyping, $5 \%$ samples were randomly selected as positive controls (repeat samples) and negative controls (without DNA template). As a result, the genotyping success rate reached $96.8 \%$, and the results of duplicated samples were $100 \%$ concordant.

\section{Statistical analysis}

All statistical tests were performed by SAS software (Version 9.4; SAS Institute, Cary, NC, USA). Differences in demographic (e.g., age and BMI) and other covariates (e.g., menarche, menopause and number of pregnancies status) between patients and controls were evaluated by Pearson's $\chi^{2}$ test. To assess the associations of these 19 SNPs with ovarian cancer risk, the adjusted odds ratios (ORs) and 95\% confidence intervals (CIs) were calculated for dominant $(\mathrm{AB}+\mathrm{BB}$ vs. $\mathrm{AA})$ and recessive models $(\mathrm{BB}$ vs. $A B+A A)$ by unconditional logistic regression analyses. A and $B$ respectively represent different alleles (wild and mutant) at this location. Additionally, stratification analysis was performed to further evaluate the significant associations existed in which demographic or clinic covariates groups. Deviation from Hardy-Weinberg Equilibrium (HWE) in the controls was assessed by a goodness-of-fit $X^{2}$ test. All $P$ value was two-sided with a significant level $<0.05$.

\section{Results}

\section{Population characteristics}

In the current study, we enrolled 196 ovarian cancer patients with an average age of $50.01 \pm 12.51$ years and 272 cancer-free controls with an average age of $50.48 \pm 11.98$ years. The demographic and clinical characteristics of all participants were shown in Supplemental Table S2. There were no significant differences between cases and controls in age $(P=0.570)$, BMI $\quad(P=0.947), \quad$ menarche $\quad(P=0.072)$, menopause $(P=0.421)$ and number of pregnancies $(P=0.448)$. To discuss the pathological classification, $132(67.4 \%), 27(13.8 \%)$ and $15(7.7 \%)$ cases were subdivided into serous, mucinous and clear cell carcinoma, respectively, while $22(11.2 \%)$ cases were included into "others" because of the limited availability of tumor samples. Regarding anatomic neoplasm subdivision, 27 (13.8\%), 74 (37.8\%) and 95 $(48.5 \%)$ ovarian cancers occurred in the left, right and both sides, respectively. In term of tumor size, 27 $(13.8 \%)$ patients reported with a tumor of $<5 \mathrm{~cm}, 74$ (37.8\%) with a tumor of $5-10 \mathrm{~cm}$ and $95(48.5 \%)$ with a tumor of $>10 \mathrm{~cm}$. According to the FIGO stage, 96 $(49.0 \%)$ and $100(51.0 \%)$ cases distributed into the stage of $\mathrm{I}+\mathrm{II}$ and $\mathrm{III}+\mathrm{V}$, respectively. Moreover, we classified all patients into four different histological grades, 14 (7.1\%) in G1, 20 (10.2\%) in G2, 114 (58.2\%) in G3+G4 and $17(8.7 \%)$ in borderline tumor, but an exception of 31 cases $(15.8 \%)$ were into the groups of "undetermined" due to the lack of information.

\section{Association between the SNPs of BER-related genes and risk of ovarian cancer}

Genotype distributions of these 19 SNPs are shown in Table 1. All these genotype distributions among the controls conformed to the HWE except FEN1 rs174538. Nevertheless, our results verified PARP rs8679 and hOGG1 rs293795 GG/AG were associated with the decreased risk of ovarian cancer under dominant model (adjusted OR $=0.39,95 \%$ $\mathrm{CI}=0.17-0.90, \quad P=0.026 ; \quad$ adjusted $\quad \mathrm{OR}=0.36, \quad 95 \%$ $\mathrm{CI}=0.13-0.99, \quad P=0.049, \quad$ respectively). And, the increased risk of ovarian cancer was observed in the participants who carried rs4796030 AA genotype compared with the genotype AC/CC (adjusted $\mathrm{OR}=1.54,95 \% \mathrm{CI}=1.01-2.35, P=0.046)$ under recessive model. However, other genetic associations with ovarian cancer risk were not discovered in the present study.

\section{Stratification analysis}

In order to further explore these significant genetic associations existing in which kinds of ovarian cancer patients, we performed a stratification analysis by age, BMI, menarche, menopause and number of pregnancies status, as well as some clinic-related factors like pathologic classification, histology grade, FIGO stage, tumor size and anatomic neoplasm subdivision (Table 2). Compared to the PARP1 rs8679 AA genotype, the protective effect of AG/GG genotypes were more predominant in the patients with BMI $<24$ (adjusted OR=0.27, 95\% CI $=0.08-0.96$, $P=0.043$ ), menarche age $\leq 14$ (adjusted OR $=0.09,95 \%$ $\mathrm{CI}=0.01-0.65, \quad P=0.017)$ and serous carcinoma (adjusted $\quad \mathrm{OR}=0.36, \quad 95 \% \quad \mathrm{CI}=0.14-0.98, \quad P=0.045$ ). Moreover, we found the LIG3 rs4796030 CC genotype carriers further increased the risk of ovarian cancer in the groups of $\mathrm{BMI} \geq 24$ (adjusted OR=2.28, 95\% 
$\mathrm{CI}=1.14-4.58, P=0.021$ ), clinical stages III/IV (adjusted $\mathrm{OR}=1.93,95 \% \mathrm{CI}=1.14-3.26, P=0.015)$ and tumor at the left (adjusted $\mathrm{OR}=2.33,95 \% \mathrm{CI}=1.34-4.06, P=0.003$ ) when compared with the AA/AC genotype carriers. Unfortunately, stratification analysis could not explain the protective effect of hOGG1 rs293795 polymorphism on which subgroup of ovarian cancer patients.

\section{Discussion}

The evidence derived from numerous studies had testified the important role of BER in the prevention of mutation accumulation [23, 24]. According to different base lesions, BER can trigger corresponding subways and assemble a series of repair complex at the site of DNA lesions [25]. Compared with other DNA repair system, the core components of BER are quite conserved across most species. As is mentioned above, the whole repair processes are simplified as three distinct phases, and the completion of each step is based on the scaffold protein of XRCC1/LIG3 and PARP1 [26]. hOGG1, one of the most important bi-functional glycosylases, is initiated by some oxidative base lesions [13]. The study reported by Xie et al. found that the hOGG1 deficiency animals would increase tumor predisposition, especially for the lung and ovarian tumors and lymphomas [27]. Similarly, with the increasing of spontaneous mutant frequencies in the APE1 heterozygous mice, the organism also suffered many deleterious effects brought by oxidative stress, including cancer [28, 29]. Although FEN1 was the only one endonuclease included in the present study, its potential role in cancer development was fully demonstrated by a heterozygous animal model [30].

Numerous investigations have tried to uncover the genetic association of ovarian cancer in these six genes; however, no firm conclusions could be drawn from them. For example, hOGG1 rs1052133 as one of the hottest spots in the recent years has been researched extensively in ovarian cancer. According to the studies of Michalska and Chen, hOGG1 rs1052133 C>G polymorphism was considered as an unfavorable factor for the susceptibility of ovarian cancer [31, 32], but a totally different result was discovered in our research. Arc and reached the same conclusion with us and suggested that there was no association between both of them [33]. Likewise, a negative association was observed between XRCC1 rs25487 G>A and ovarian cancer risk in Serbian women [34], while this finding was unable to repeat in another study by Khokhrin and ours [35]. In fact, these contradictory conclusions were caused by several factors, of which the relatively limited sample size and different genetic backgrounds a probably the most critical reasons.

In this current hospital-based case-control study, we not only explored the impact of 19 potentially functional SNPs in six BER-related genes on ovarian cancer risk, but also conducted an analysis of gene-environmental interactions in a certain number of cases and controls from two different institutions.

Table 1. Association between polymorphisms in base excision repair pathway gene and ovarian cancer risk

\begin{tabular}{|c|c|c|c|c|c|c|c|c|c|c|c|c|c|c|}
\hline \multirow[t]{2}{*}{ Gene } & \multirow[t]{2}{*}{ SNP } & \multicolumn{2}{|c|}{ Allele } & \multicolumn{3}{|c|}{ Case $(\mathrm{N}=196)$} & \multicolumn{3}{|c|}{ Control $(\mathrm{N}=272)$} & \multirow{2}{*}{$\begin{array}{l}\text { Adjusted ORa } \\
(95 \% \mathrm{CI})\end{array}$} & \multirow[t]{2}{*}{$P a$} & \multirow{2}{*}{$\begin{array}{l}\text { Adjusted OR }{ }^{\mathrm{b}} \\
(95 \% \mathrm{CI})\end{array}$} & \multirow[t]{2}{*}{$P \mathrm{~b}$} & \multirow[t]{2}{*}{ HWE } \\
\hline & & $\mathrm{A}$ & $\mathrm{B}$ & AA & $\mathrm{AB}$ & $\mathrm{BB}$ & AA & $\mathrm{AB}$ & BB & & & & & \\
\hline PARP1 & rs2666428 & $\mathrm{T}$ & $\mathrm{C}$ & 129 & 49 & 7 & 171 & 87 & 13 & $0.73(0.49-1.09)$ & 0.126 & $0.80(0.31-2.05)$ & 0.637 & 0.653 \\
\hline PARP1 & rs8679 & A & G & 177 & 8 & 0 & 241 & 26 & 1 & $0.39(0.17-0.90)$ & 0.026 & - & - & 0.740 \\
\hline hOGG1 & rs1052133 & G & $\mathrm{C}$ & 78 & 87 & 29 & 110 & 115 & 47 & $1.03(0.71-1.51)$ & 0.870 & $0.84(0.50-1.39)$ & 0.486 & 0.079 \\
\hline hOGG1 & rs159153 & $\mathrm{T}$ & $\mathrm{C}$ & 160 & 32 & 1 & 221 & 46 & 5 & $0.95(0.58-1.54)$ & 0.823 & $0.28(0.03-2.46)$ & 0.252 & 0.164 \\
\hline hOGG1 & rs293795 & $\mathrm{A}$ & G & 183 & 5 & 0 & 253 & 18 & 1 & $0.36(0.13-0.99)$ & 0.049 & - & - & 0.279 \\
\hline FEN1 & rs174538 & G & A & 45 & 143 & 0 & 73 & 199 & 0 & $1.17(0.76-1.79)$ & 0.484 & - & - & $<0.001$ \\
\hline FEN1 & rs4246215 & G & $\mathrm{T}$ & 46 & 96 & 52 & 74 & 135 & 63 & $1.22(0.80-1.88)$ & 0.361 & $1.26(0.82-1.93)$ & 0.296 & 0.925 \\
\hline APEX1 & rs1130409 & $\mathrm{T}$ & G & 64 & 100 & 29 & 91 & 123 & 54 & $1.05(0.71-1.57)$ & 0.801 & $0.72(0.43-1.18)$ & 0.190 & 0.293 \\
\hline APEX1 & rs1760944 & $\mathrm{T}$ & G & 65 & 89 & 38 & 104 & 122 & 46 & $1.18(0.80-1.74)$ & 0.412 & $1.18(0.73-1.91)$ & 0.501 & 0.321 \\
\hline APEX1 & rs3136817 & $\mathrm{T}$ & $\mathrm{C}$ & 158 & 33 & 3 & 224 & 43 & 5 & $1.08(0.67-1.75)$ & 0.754 & $0.85(0.20-3.64)$ & 0.822 & 0.095 \\
\hline LIG3 & rs1052536 & $\mathrm{C}$ & $\mathrm{T}$ & 92 & 85 & 15 & 131 & 116 & 21 & $1.04(0.71-1.51)$ & 0.847 & $1.02(0.51-2.05)$ & 0.958 & 0.502 \\
\hline LIG3 & rs3744356 & $\mathrm{C}$ & $\mathrm{T}$ & 188 & 3 & 1 & 261 & 10 & 0 & $0.56(0.17-1.82)$ & 0.331 & - & - & 0.757 \\
\hline LIG3 & rs4796030 & A & $\mathrm{C}$ & 77 & 55 & 61 & 88 & 117 & 62 & $0.73(0.49-1.08)$ & 0.112 & $1.54(1.01-2.35)$ & 0.046 & 0.060 \\
\hline XRCC1 & rs1799782 & G & A & 99 & 75 & 19 & 146 & 105 & 18 & $1.11(0.77-1.62)$ & 0.570 & $1.53(0.78-3.01)$ & 0.218 & 0.881 \\
\hline XRCC1 & rs25487 & $\mathrm{C}$ & $\mathrm{T}$ & 89 & 83 & 21 & 146 & 97 & 24 & $1.43(0.98-2.08)$ & 0.063 & $1.31(0.70-2.45)$ & 0.395 & 0.182 \\
\hline XRCC1 & rs25489 & G & A & 139 & 50 & 5 & 215 & 50 & 7 & $1.48(0.96-2.28)$ & 0.074 & $0.99(0.31-3.21)$ & 0.985 & 0.059 \\
\hline XRCC1 & rs2682585 & G & A & 146 & 44 & 3 & 205 & 60 & 3 & $1.06(0.68-1.64)$ & 0.803 & $1.49(0.29-7.62)$ & 0.634 & 0.547 \\
\hline XRCC1 & rs3810378 & G & $\mathrm{C}$ & 102 & 70 & 23 & 147 & 101 & 24 & $1.10(0.76-1.60)$ & 0.624 & $1.45(0.79-2.66)$ & 0.236 & 0.273 \\
\hline XRCC1 & rs915927 & $\mathrm{T}$ & $\mathrm{C}$ & 159 & 33 & 1 & 213 & 56 & 3 & $0.76(0.47-1.22)$ & 0.261 & $0.49(0.05-4.87)$ & 0.543 & 0.749 \\
\hline
\end{tabular}

OR, odds ratio; $\mathrm{CI}$, confidence interval. HWE, Hardy-Weinberg equilibrium.

The results were in bold, if the $95 \% \mathrm{CI}$ excluded 1 or $P$-values less than 0.05 .

a: Adjusted for age, BMI, menarche, menopause, number of pregnancies for dominant model.

$\mathrm{b}$ : Adjusted for age, BMI, menarche, menopause, number of pregnancies for recessive model. 
Table 2. Stratification analysis of base excision repair pathway gene variant genotypes with ovarian cancer risk

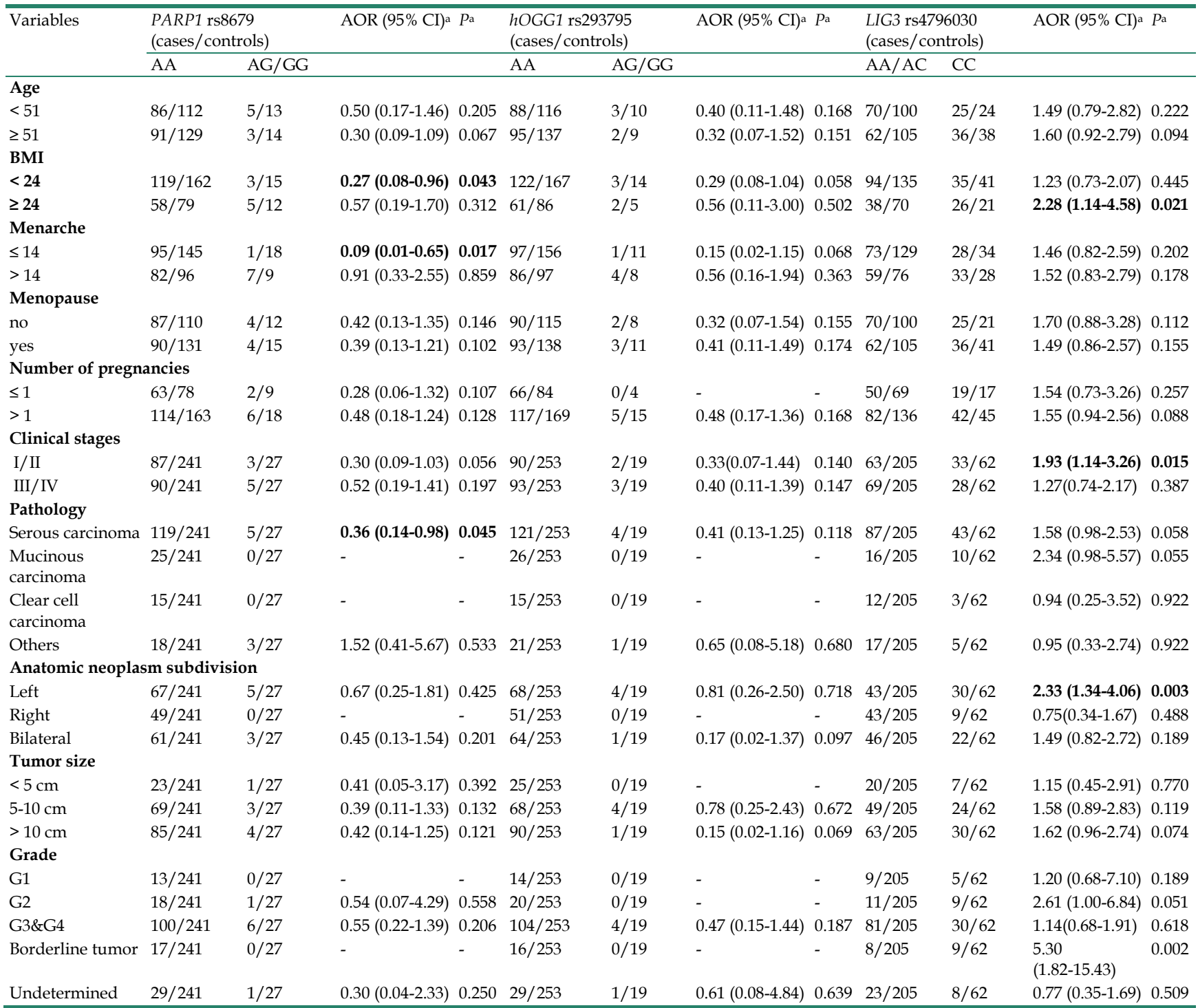

CI, confidence interval; AOR, adjusted odds ratio.

The results were in bold, if the $95 \%$ CI excluded 1 or $P$ values less than 0.05

a: Obtained in logistic regression models with adjustment for age, BMI, menarche, menopause, number of pregnancies.

The genotyping results indicated that the PARP1 rs8679 and hOGG1 rs293795 A>G polymorphisms were associated with the decreased ovarian cancer risk under a dominant model, while LIG3 rs4796030 A $>$ C polymorphism with an increased ovarian cancer risk under a recessive model. Additionally, we noticed that the protective effect of PARP1 rs8679 $A>G$ polymorphism was more pronounced among the groups of $\mathrm{BMI}<24$, menarche age $\leq 14$ and serous carcinoma. Moreover, the negative effect of LIG3 rs4796030 A $>C$ polymorphism significantly associated with the patients in the groups of $B M I \geq 24$, clinical stages III/IV and tumor at the left. The preliminary results obtained here indicated that compared with patients of $\mathrm{BMI} \geq 24$, patients of $\mathrm{BMI}<$ $24 \mathrm{had}$ a more prominent protective effect of genetic variation, and their negative effects were relatively small. These results showed that healthy lifestyle also played an important role in the development of cancer. The role of genetic variation was also related to the pathological type and clinical stage of cancer.

Although we found two novel related SNPs associating with the risk of ovarian cancer, some inherent limitations still should be listed to discussion. Firstly, the limited number of cases and controls would cause a deficiency of statistical power to some extent. Secondly, many relative risk factors of ovarian cancer, such as oral contraceptive, CA125 and breast feeding, are not fully considered due to lack of individual information. Thirdly, only 19 SNPs of six BER-related genes were investigated in the present study, which was insufficiently to explain the 
biological relationship of BER with the risk of ovarian cancer. Fourthly, we noticed that the genotype frequencies of FEN1 rs174538 G>A deviate from HWE, which indicates that our research may exist the problem sampling bias to some extent. Finally, these new findings in our research were not confirmed in vitro and in vivo experiences, and the survival analysis for SNPs was not explored further.

In conclusion, our study suggested that the PARP1 and hOGG1 polymorphisms might correlate to ovarian cancer susceptibility. However, more comprehensive studies with larger independent cohorts should be provided to verify the relationship between these significant genetic variations in PARP1 and $h O G G 1$ gens and ovarian cancer risk.

\section{Supplementary Material}

Supplementary tables.

http://www.jcancer.org/v12p0264s1.pdf

\section{Acknowledgements}

This study was supported by grants from Lin He's New Medicine and Clinical Translation Academician Workstation Research Fund (17331204), Zhejiang Provincial Medical and Health Science and Technology plan (2018ZD009), the Major Science and Technology Special Project of Wenzhou Science and Technology Bureau (ZY2020009) and Le Fund (KH-2020-LJJ-030).

\section{Competing Interests}

The authors have declared that no competing interest exists.

\section{References}

1. Bray F, Ferlay J, Soerjomataram I, Siegel RL, Torre LA, Jemal A. Global cancer statistics 2018: GLOBOCAN estimates of incidence and mortality worldwide for 36 cancers in 185 countries. CA Cancer J Clin. 2018; 68: 394-424.

2. Modugno F. Ovarian cancer and high-risk women-implications for prevention, screening, and early detection. Gynecol Oncol. 2003; 91: 15-31.

3. Nunns D, Symonds P, Ireland D. Surgical management of advanced ovarian cancer. Obstet Gynecol Surv. 2000; 55: 746-51.

4. Schwartz MD, Kaufman E, Peshkin BN, Isaacs C, Hughes C, DeMarco T, et al. Bilateral prophylactic oophorectomy and ovarian cancer screening following BRCA1/BRCA2 mutation testing. J Clin Oncol. 2003; 21: 4034-41.

5. Mostowska A, Pawlik P, Sajdak S, Markowska J, Pawałowska M, Lianeri M, et al. An Analysis of Polymorphisms Within the Wnt Signaling Pathway in Relation to Ovarian Cancer Risk in a Polish Population. Mol Diagn Ther. 2014; 18(1): 85-91.

6. Zhao Z, Zhang A, Zhao Y, Xiang J, Yu D, Liang Z, et al. The association of polymorphisms in nucleotide excision repair genes with ovarian cancer susceptibility. Biosci Rep. 2018; 38: undefined.

7. He J, Zhuo ZJ, Zhang A, Zhu J, Hua RX, Xue WQ, et al. Genetic variants in the nucleotide excision repair pathway genes and gastric cancer susceptibility in a southern Chinese population. Cancer Manag Res. 2018; 10: 765-74.

8. Chatterjee N, Walker GC. Mechanisms of DNA damage, repair, and mutagenesis. Environ Mol Mutagen. 2017; 58: 235-63.

9. Hitomi K, Iwai S, Tainer JA. The intricate structural chemistry of base excision repair machinery: implications for DNA damage recognition, removal, and repair. DNA Repair. 2007; 6: 410-28.

10. Zhou PT, Li B, Ji J, Wang MM, Gao CF. A systematic review and meta-analysis of the association between OGG1 Ser326Cys polymorphism and cancers. Med Oncol. 2015; 32: 472.
11. Almeida KH, Sobol RW. A unified view of base excision repair: lesion-dependent protein complexes regulated by post-translational modification. DNA repair. 2007; 6: 695-711.

12. Wood RD, Mitchell M, Sgouros J, Lindahl T. Human DNA repair genes. Science. 2001; 291: 1284-9.

13. Hazra TK, Hill JW, Izumi T, Mitra S. Multiple DNA glycosylases for repair of 8-oxoguanine and their potential in vivo functions. Prog Nucleic Acid Res Mol Biol. 2001; 68: 193-205.

14. Demple B, Sung JS. Molecular and biological roles of Ape1 protein in mammalian base excision repair. DNA repair. 2005; 4: 1442-9.

15. Caldecott KW, Aoufouchi S, Johnson P, Shall S. XRCC1 polypeptide interacts with DNA polymerase beta and possibly poly (ADP-ribose) polymerase, and DNA ligase III is a novel molecular 'nick-sensor' in vitro. Nucleic Acids Res. 1996; 24: 4387-94.

16. Dianova II, Bohr VA, Dianov GL. Interaction of human AP endonuclease 1 with flap endonuclease 1 and proliferating cell nuclear antigen involved in long-patch base excision repair. Biochemistry. 2001; 40: 12639-44.

17. Rice PA. Holding damaged DNA together. Nat Struct Biol. 1999; 6: 805-6.

18. Marintchev A, Mullen MA, Maciejewski MW, Pan B, Gryk MR, Mullen GP. Solution structure of the single-strand break repair protein XRCC1 N-terminal domain. Nat Struct Biol. 1999; 6: 884-93.

19. Zhu J, Jia W, Wu C, Fu W, Xia H, Liu G, et al. Base Excision Repair Gene Polymorphisms and Wilms Tumor Susceptibility. EBioMedicine. 2018; 33: 88-93.

20. He J, Wang F, Zhu J, Zhang R, Yang T, Zou Y, et al. Association of potentially functional variants in theXPGgene with neuroblastoma risk in a Chinese population. J Cell Mol Med. 2016; 20: 1481-90.

21. He J, Zou Y, Wang T, Zhang R, Yang T, Zhu J, et al. Genetic Variations of GWAS-Identified Genes and Neuroblastoma Susceptibility: a Replication Study in Southern Chinese Children. Transl Oncol. 2017; 10: 936-41.

22. He J, Zou Y, Liu X, Zhu J, Zhang J, Zhang R, et al. Association of Common Genetic Variants in Pre-microRNAs and Neuroblastoma Susceptibility: A Two-Center Study in Chinese Children. Mol Ther Nucleic Acids. 2018; 11: 1-8.

23. Lindahl T. Instability and decay of the primary structure of DNA. Nature. 1993; 362: 709-15.

24. Lindahl T, Wood RD. Quality control by DNA repair. Science. 1999; 286: 1897-905.

25. Fan J, Wilson DM. Protein-protein interactions and posttranslational modifications in mammalian base excision repair. Free Radic Biol Med. 2005; 38: 1121-38.

26. Masson M, Niedergang C, Schreiber V, Muller S, Menissier-de Murcia J, de Murcia G. XRCC1 is specifically associated with poly(ADP-ribose) polymerase and negatively regulates its activity following DNA damage. Mol Cell Biol. 1998; 18: 3563-71.

27. Xie Y, Yang H, Cunanan C, Okamoto K, Shibata D, Pan J, et al. Deficiencies in Mouse Myh and Ogg1 Result in Tumor Predisposition and G to T Mutations in Codon 12 of the K-ras Oncogene in Lung Tumors. Cancer Res. 2004; 64: 3096-102.

28. Huamani J, McMahan CA, Herbert DC, Reddick R, McCarrey JR, MacInnes MI, et al. Spontaneous mutagenesis is enhanced in Apex heterozygous mice. Mol Cell Biol. 2004; 24: 8145-53.

29. Tell G, Damante G, Caldwell D, Kelley MR. The intracellular localization of APE1/Ref-1: more than a passive phenomenon? Antioxid Redox Signal. 2005; 7: 367-84.

30. Kucherlapati M, Yang K, Kuraguchi M, Zhao J, Lia M, Heyer J, et al Haploinsufficiency of Flap endonuclease (Fen1) leads to rapid tumor progression. P Natl Acad Sci USA. 2002; 99: 9924-9.

31. Michalska MM, Samulak D, Romanowicz H, Bieńkiewicz J, Sobkowski M, Ciesielski K, et al. Single nucleotide polymorphisms (SNPs) of hOGG1 and XRCC1 DNA repair genes and the risk of ovarian cancer in Polish women. Tumour Biol. 2015; 36: 9457-63.

32. Chen X, Liu X, Wang J, Guo W, Sun C, Cai Z, et al. Functional polymorphisms of the hOGG1 gene confer risk to type 2 epithelial ovarian cancer in Chinese. Int J Gynecol Cancer. 2011; 21: 1407-13.

33. Arcand SL, Provencher D, Mes-Masson AM, Tonin PN. OGG1 Cys326 variant, allelic imbalance of chromosome band 3p25.3 and TP53 mutations in ovarian cancer. Int J Oncol. 2005; 27: 1315-20.

34. Malisic EJ, Krivokuca AM, Boljevic IZ, Jankovic RN. Impact of RAD51 G135C and XRCC1 Arg399Gln polymorphisms on ovarian carcinoma risk in Serbian women. Cancer Biomark. 2015; 15: 685-91.

35. Khokhrin DV, Khrunin AV, Moiseev AA, Gorbunov VA, Limborskaia SA. Association of polymorphisms in glutathione-S-transferase and DNA repair genes with ovarian cancer risk in the Russian population. Genetika. 2012; 48: $901-4$ 